\title{
Démarrage en pompe des groupes réversibles - Différents procédés utilisables. Avantages et inconvénients.
}

\author{
par 6. Martin \\ Chef de Division, Service Matériel, R.E.H. Alpes-Nord, \\ E.D.F., Chambéry \\ Rapporteur du Groupe de travail "Schémas de démarrage des groupes réversibles"
}

\section{1. - Généralités sur le démarrage en pompe}

Par rapport aux groupes ternaires utilisant deux machines distinctes classiques: une pompe et une turbine, les groupes réversibles pompes-turbines permettent de réaliser d'importantes économies sur les frais d'investissement des usines d'accumulation d'énergie par pompage, en particulier, dans le cas où les puissances demandées en turbine et en pompe ne sont pas très différentes et lorsque la rapidité du passage d'une marche à l'autre n'est pas un facteur déterminant.

En contrepartie, ces machines réversibles, à double sens de rotation, posent un certain nombre de problèmes, dont l'un des plus difficiles à résoudre, surtout pour des unités de forte puissance, est le lancement du groupe dans le sens «pompage».

De nombreux procédés sont possibles. Ils peuvent se classer très schématiquement comme indiqué sur le tableau de la figure 1 . Le choix de l'un d'eux dépend de nombreux facteurs de sorte qu'il doit faire, chaque fois, l'objet d'une étude particulière.

Au niveau de la turbine deux possibilités s'offrent au projeteur : démarrage avec roue noyée ou avec roue dénoyée.

La première solution est évidemment la plus simple et la plus rapide. Elle conduit à une prise de charge régulière et progressive, mais elle impose un moyen de démarrage important, capable de vaincre le couple de barbotage de la roue. Peu de procédés admettent le démarrage roue noyée, tout au moins pour des machines d'une certaine puissance.

La deuxième solution demande une installation importante de dénoyage de la roue par air comprimé et un dispositif d'arrosage des labyrinthes; le renoyage de la roue par évacuation de la poche d'air, après synchronisation au réseau, est délicat. Un à-coup plus ou moins brutal est inévitable au moment où le niveau d'eau atteint la base de la roue. Cet à-coup se répercute sur la machine électrique et sur le réseau. L'installation d'air comprimé est onéreuse et encombrante. Toutefois, dans certains cas, elle peut être valorisée par la possibilité qu'elle offre de marche du groupe en compensateur synchrone.
Pour les groupes de forte puissance, la plupart des procédés de démarrage imposent pratiquement le démarrage roue dénoyée.

Les courbes de la figure 2 montrent l'allure générale du couple résistant d'une pompe-turbine en fonction de la vitesse pour les deux cas: roue noyée et roue dénoyée.

Ces courbes font, également, ressortir l'intérêt de l'injection d'huile au pivot qui permet de réduire, dans des
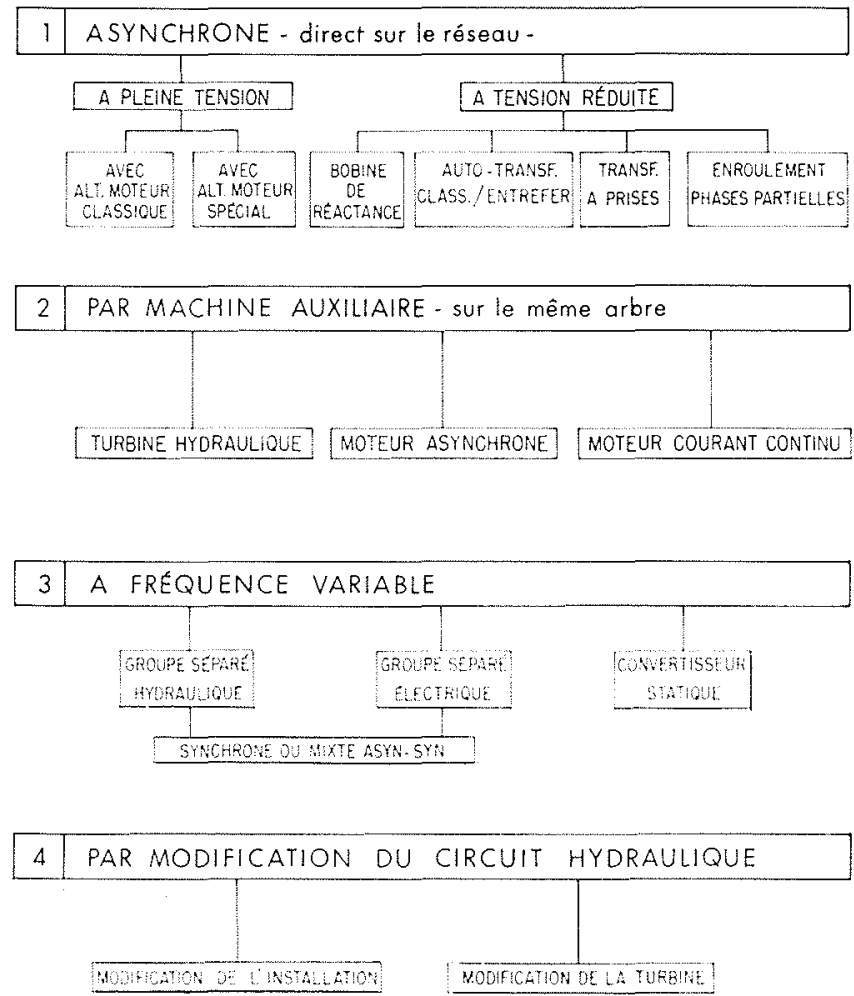

1/ Procédés de lancement des groupes réversibles. 


\section{G. MARTIN}

proportions importantes, le couple de décollage et par conséquent le couple initial de démarrage du moteur.

Le choix entre les deux solutions: roue noyée et roue dénoyée est principalement fonction des possibilités de couple qu'offre la méthode de lancement choisie.

Dans tous les cas, après couplage au réseau, la charge est prise par ouverture de la vanne de refoulement ou du distributeur mobile, si la machine en est munie.

Rappelons également que, quel que soit le procédé, sauf cas particulier de la turbine isogyre (*), il faut réaliser une inversion de phases du réseau entre les marches en turbine et en pompe. Cette inversion peut se faire côté haute tension ou côté moyenne tension du groupe.

Pour les groupes de forte puissance, il peut être intéressant de faire l'inversion côté haute tension, ou sur la ligne, dans les usines à plusieurs groupes, la marche simultanée de certains groupes en pompage et d'autres en turbinage étant évidemment exclue. Cette solution n'est pas applicable, s'il existe des soutirages pour des auxiliaires au niveau moyenne tension.

Dans d'autres cas et notamment dans le cas d'un schéma avec un appareillage de démarrage commun à plusieurs groupes (bobines de réactance, auto-transformateur, etc.), il peut au contraire être avantageux de créer un jeu de barres, moyenne tension, au niveau duquel se fera l'inversion.

Pour simplifier, dans les schémas suivants, nous avons adopté la disposition: groupe-bloc avec commutation côté moyenne tension. Mais d'autres formes de schéma pourraient être admises, par exemple avec barres de transfert pour les appareillages communs à plusieurs groupes ou avec commutation côté haute tension. De niême, les dispositifs d'excitation, variables suivant les cas et suivant les constructeurs, n'ont pas été détaillés.

Enfin, seuls les appareillages essentiels indispensables à la compréhension du processus ont été représentés, à l'exclusion des appareillages secondaires classiques tels que les sectionneurs d'isolement.

Les divers procédés utilisables pour le lancement en pompe des groupes réversibles sont examinés ci-après, en mentionnant pour chacun d'eux les principaux avantages et inconvénients.

\section{2. - Démarrage asynchrone direct sur le réseau}

Dans ce mode de lancement, l'alternateur-moteur à l'arrêt et non excité est brusquement couplé au réseau, soit directement sous la pleine tension, soit par l'intermédiaire d'un appareillage spécial destiné à réduire la tension aux bornes de l'alternateur pour limiter le courant de démarrage et la chute de tension qui en résulte, à des valeurs acceptables pour le réseau.

Tous ces procédés ont, en commun, l'avantage de la très grande simplicité du schéma et de la rapidité de démarrage. Par contre, ils présentent un certain nombre d'inconvénients:

- appel de courant sur le réseau très important pouvant se traduire par une chute de tension inacceptable pour les exploitants;

- énergie thermique à évacuer dans les masses polaires pendant la mise en vitesse considérable, ce qui impose une construction spéciale des rotors: circuits amortisseurs à cage fermée complète avec, éventuellement, circulation d'eau de refroidissement (surtout pour les ma-

(*) Brevet Ateliers des Charmilles. chines à démarrages fréquents), emploi de matériaux électriquement plus résistants, tels que laiton ou bronze, remplacement des pôles feuilletés par des pôles massifs avec anneaux amortisseurs, etc.;

- intervalle de temps, entre deux démarrages, imposé par des considérations de refroidissement des pôles;

- efforts électro-dynamiques importants dans les bobinages du stator et du transformateur de couplage, d'où la nécessité d'une étude très poussée du calage et de la résistance mécanique des bobinages;

- impossibilité, en pratique, de démarrer simultanément tous les groupes d'une même usine (mais ceci est un inconvénient propre à presque tous les procédés).

\subsection{Démarrage direct à pleine tension.}

Le schéma, très simple, est donné par la figure 3.

Le processus est le suivant: après dénoyage de la roue et injection d'huile au pivot, presque toujours indispensable dans ce cas, le moteur, non excité, est couplé au réseau par fermeture du disjoncteur H.T. La machine démarre et lorsque le rotor atteint son régime asynchrone permanent, il est synchronisé par enclenchement de l'excitation. La roue est renoyée et la charge est prise par ouverture du vannage ou de la vanne de refoulement.

Aux avantages techniques déjà signalés s'ajoutent ceux de l'économie, puisque aucun appareillage spécial est nécessaire.

Le quatrième groupe de la centrale de Revin, d'une puissance de $200 \mathrm{MW}$ avec rotor à amortisseurs réfrigérés par eau, sera démarré selon ce procédé.

\subsection{Démarrage à tension réduite.}

L'inconvénient principal du démarrage à pleine tension, inconvénient d'où d'ailleurs découlent tous les autres, est l'importance considérable de l'appel de courant sur le réseau et des chutes de tension qui en découlent.

Outre le dénoyage de la roue, divers moyens ont été proposés pour limiter cette pointe de courant. Ils ont tous pour but d'augmenter la réactance globale du circuit statorique, pendant la période de mise en vitesse.

L'à-coup de courant est diminué mais, en contrepartie, le démarrage est plus lent, d'autant plus lent que la limitation a été plus énergique.

Plusieurs moyens peuvent être utilisés pour démarrer à tension réduite:

\subsubsection{DÉmarRage AVEC BoBINe de RÉACTANCE.}

Une bobine de réactance est insérée, pendant le démarrage en série, dans le circuit du moteur, côté phase ou côté neutre, suivant les schémas des figures $4 a$ et $4 b$.

Au cours de la mise en vitesse du groupe, l'impédance apparente de l'alternateur augmente, tandis que celle de la bobine reste constante, de telle sorte que la tension qui se répartit proportionnellement aux impédances crôt progressivement aux bornes du moteur. Le couple moteur-asynchrone a une caractéristique légèrement ascendante, ce qui est favorable.

A l'égalité du couple moteur et du couple résistant, la machine est synchronisée par fermeture de l'excitation. La bobine de réactance est alors court-circuitée pour achever le démarrage; cette manœuvre s'accompagne d'un bref à-coup, plus ou moins important, suivant l'écart des vitesses, mais généralement admissible.

La même bobine peut être utilisée pour démarrer, successivement, plusieurs groupes identiques. Elle doit être alors calculée pour supporter plusieurs démarrages successils. 
(A) Couple avec roue noyée et injection d'huile sous le pivot

(B) " " "sans " " "

(C) Couple avec roue dénoyée et injection d'huile sous le pivot

(D)

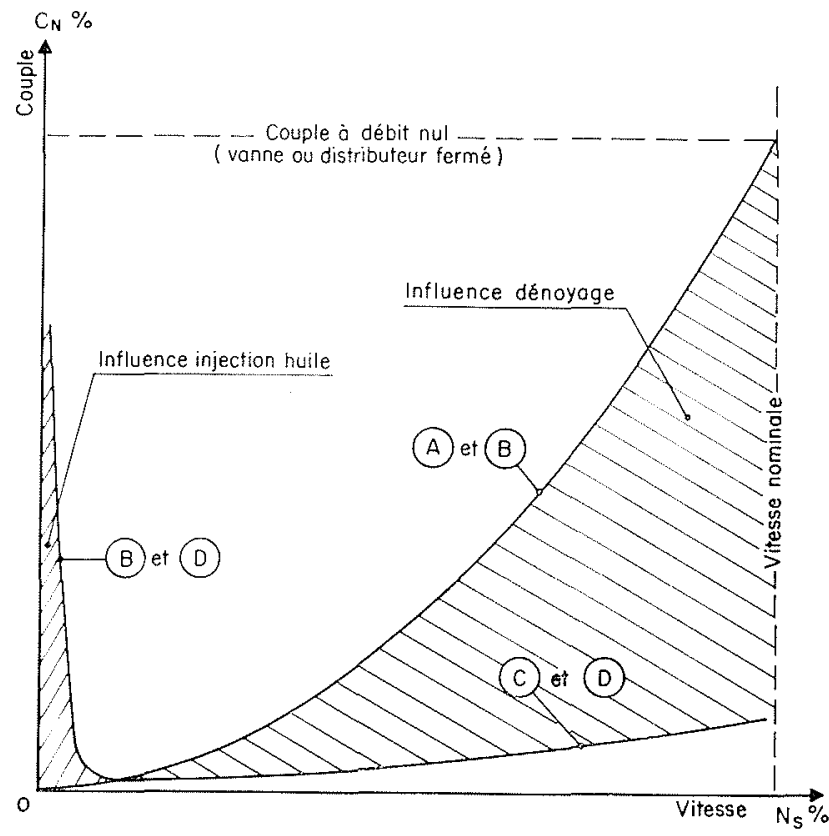

2/

2/ Allure du couple résistant en fonction de la vitesse.

3/ Démarrage en asynchrone. Démarrage direct à pleine tension.

4 a/ Démarrage en asynchrone avee tension réduite par des bobines de réactance insérées côté neutre.

$\mathbf{4 b}$ / Démarrage en asynchrone avec tension réduite par des bobines de réactance insérées côté phase.

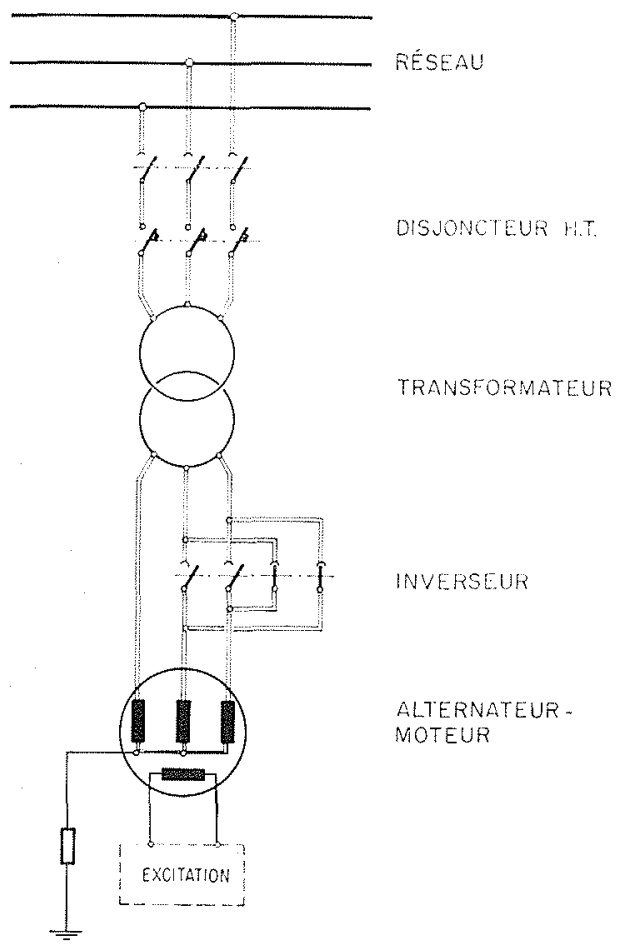

RÉSEAU

DISJONCTEUR H.T.

TRANSFORMATEUR

INVERSEUF

ALTERNATEUR MOTEUR

COURT-CIRCUITEUR

RÉACTANCES

4 al

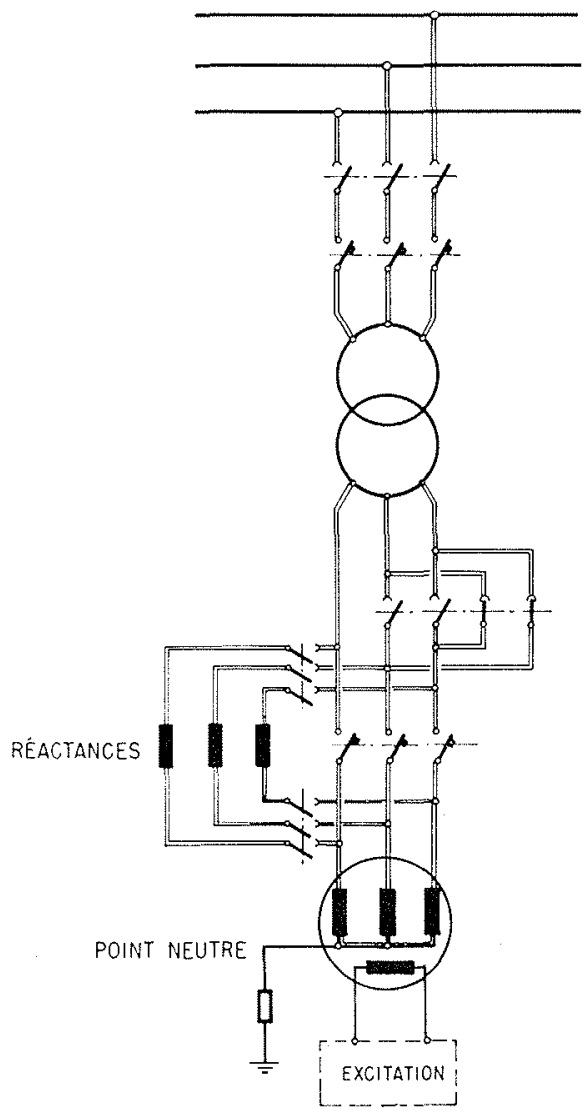

RÉSEAU

DISJONCTEUR H.T.

TRANSFORMATEUR

NVERSEUR

COURT - CIRCUITEUR

ALTERNATEUR MOTEUR 


\section{G. MARTIN}

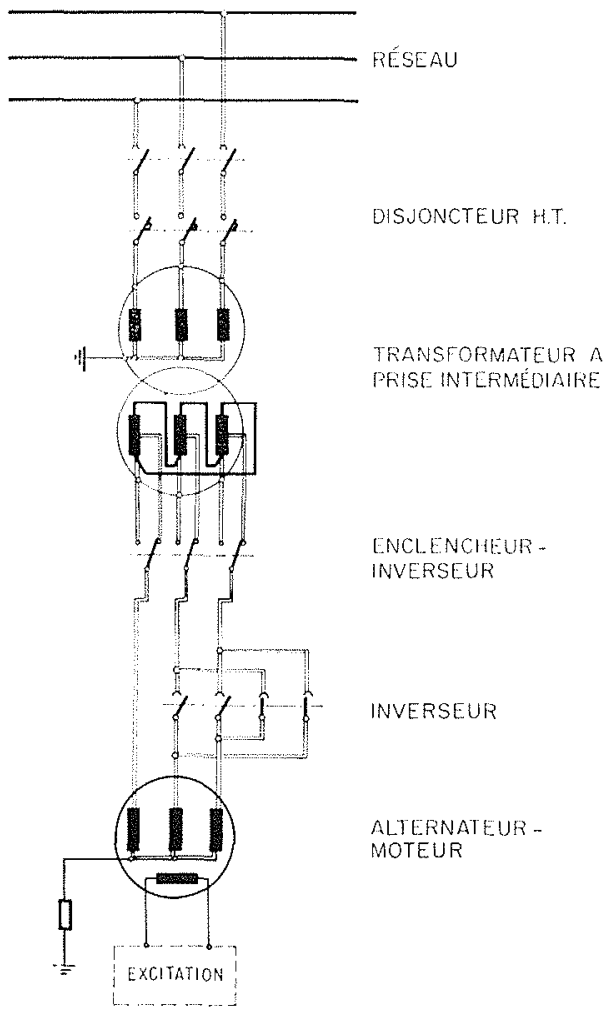

5/ Démarrage en asynchrone avec transformateur à prise intermédiaire.

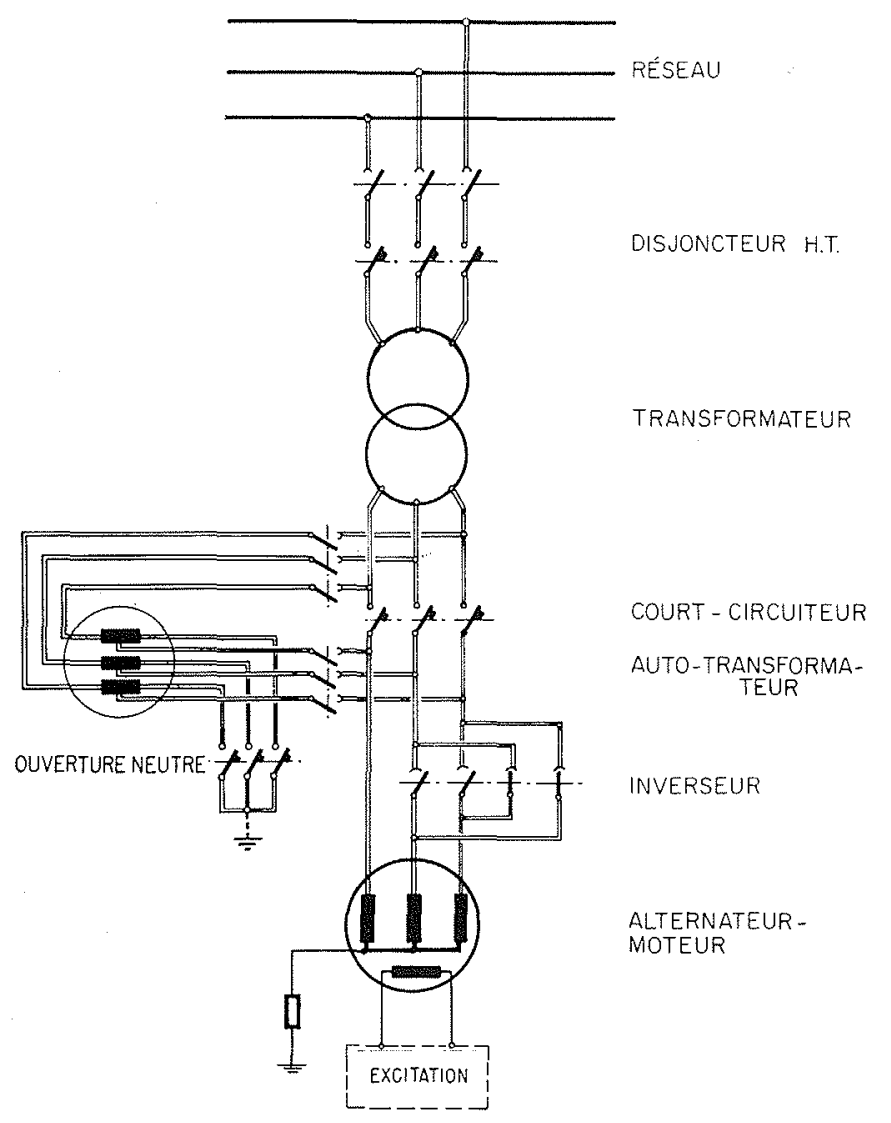

6/ Démarrage en asynchrone avec tension réduite par un autotransformateur.
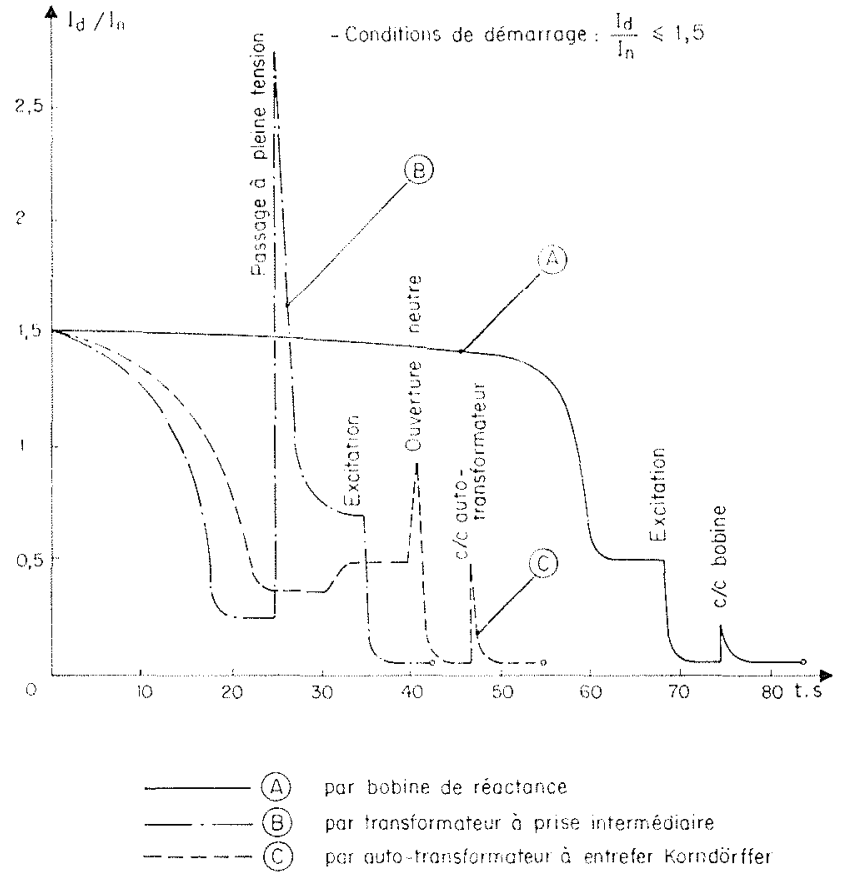

7/ Démarrage en asynchrone avec tension réduite - (d'après B.B.C.)

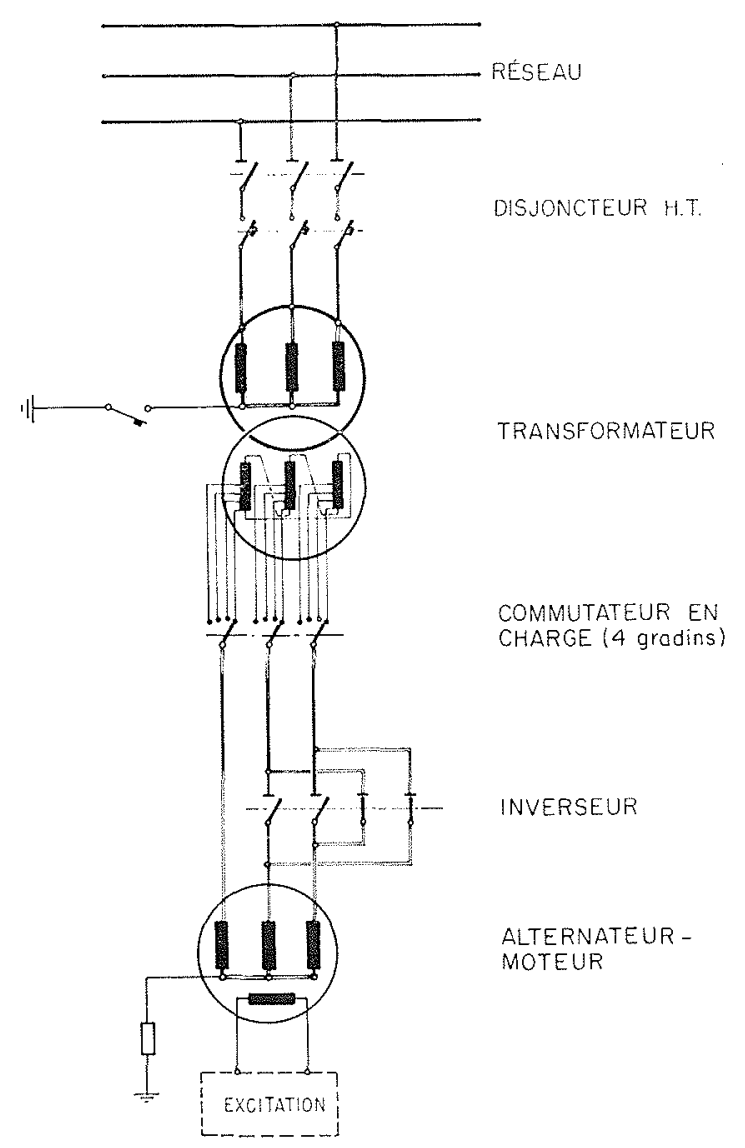

8/ Démarrage en asynchrone avec transformateur à gradins. 
La méthode est plus onéreuse que le démarrage direct, en raison du coût non négligeable de la réactance (surtout pour les usines ne comportant qu'un ou deux groupes), mais elle reste simple; la réactance, organe statique, ne demandant aucun entretien particulier et les coupleurs pour très fortes intensités étant, maintenant, bien au point.

L'à-coup de courant au démarrage étant limité, les inconvénients qui en découlent sont réduits. Si la limitation de courant choisie n'est pas trop grande, la mise en vitesse reste rapide.

Par contre, au démarrage le $\cos \varnothing$ est assez bas $(0,15$ environ) et le couple est plus faible. Si le réseau impose une réduction de l'intensité de démarrage dans le rapport $\mathrm{K}$, le couple diminue dans le rapport $\mathrm{K}^{2}$. Le démarrage risque d'être beaucoup plus long.

Le groupe $n^{0} 10$ de Vianden (Luxembourg) de $210 \mathrm{MW}$ utilisera ce procédé de démarrage.

\subsubsection{DÉMARRAGE AVEC TRANSFORMATEUR A PRISE INTER- MÉDIATRE.}

La réduction de tension est obtenue par une prise intermédiaire sur l'enroulement M.T. du transfomateur principal suivant figure 5 .

Au démarrage, l'inverseur permet de ne prélever qu'une partie de la tension et le moteur démarre en asynchrone. Le glissement stationnaire étant atteint, la peine tension est appliquée au moteur par basculement de l'inverseur. Le rotor passe en régime asynchrone permanent, il est excité et synchronisé au réseau.

La mise en vitesse est rapide car, dans ce cas, pour une réduction de courant côté réseau dans le rapport $K$, le couple-moteur n'est plus réduit que dans le rapport $\mathrm{K}$ également. Par contre, l'à-coup de courant au passage à pleine tension est très important et peut ne pas être admis par le réseau.

Comme dans le cas précédent, le procédé est économique et sans sujétions d'entretien.

\subsubsection{DÉmARRAGE AVEC AUTO-TRANSFORMATEUR}

Un auto-transformateur est inséré, côté phase, entre le transformateur principal et le moteur comme représenté par la figure $\mathrm{n}^{\circ} 6$.

Le processus de démarrage reste le même. Le court-circuitage de l'auto-transformateur par l'enclencheur donne, également, un à-coup de courant non négligeable, mais que l'on peut limiter en ouvrant le neutre de l'auto-transformateur un peu avant le court-circuitage. Pendant quelques instants, celui-ci se comporte comme une bobine de réactance, de très faible valeur il est vrai, en série avec le moteur mais qui contribue à limiter légèrement l'appel de courant. Le neutre peut ou non être mis à la terre.

Ce procédé est analogue au précédent, dont il présente sensiblement les mêmes avantages et les mêmes inconvénients, mais il n'est économiquement valable que pour les moteurs de faible puissance et pour une usine à plusieurs groupes, l'auto-transformateur étant alors commun à tous les groupes.

L'inconvénient de l'à-coup de courant peut être limité en utilisant un auto-transformateur spécial à entrefer du type Korndörfer, dont la réactance, élevée, contribue à limiter la pointe de courant.

Les courbes du cliché 7 (d'après B.B.C.) montrent, à titre indicatif, l'évolution du courant en fonction du temps pour la même machine et pour la même hypothèse de limitation du courant absorbé, pour les trois procédés, ci-dessus, de démarrage à tension réduite.

\subsubsection{DÉmaRRAge AVEC TRANSFORMATEuR A GRADINS.}

Ce procédé - variante du démarrage par transformateur à prise - consiste à faire varier la tension aux bornes du moteur au cours du démarrage, non pas en une seule fois, mais par gradins succesifs $(4,6$, ou plus) entre une valeur minimale et la valeur nominale, en l'ajustant au mieux en fonction du couple résistant.

Un choix judicieux de nombre de gradins et de leur instant de commutation permet d'obtenir un minimum de perturbation sur le réseau.

Par contre, outre l'affaiblissement du bobinage du transformateur, l'inconvénient majeur de ce dispositif réside dans la fiabilité des commutateurs en charge qui sont des organes d'une technologie délicate, surtout lorsque les courants prennent quelque importance (au-delà de 7000 à $8000 \mathrm{~A}$ ).

La figure 8 représente le schéma d'une telle installation.

\subsubsection{DÉmarrage a TENSION RÉdUITE AVEC FRACTIONNE- MENT DE L'ENROULEMENT STATORIQUE (OU A PHASES PARTIELLES).}

Les quatre procédés précédents font appel à des dispositions d'augmentation artificielle de la réactance du circuit statorique à l'extérieur de l'alternateur-moteur. Il est possible, également, d'augmenter la réactance interne de la machine, mais uniquement pendant la phase asynchrone du démarrage en n'utilisant, par exemple, qu'une partie de l'enroulement.

Le principe consiste à diviser l'enroulement statorique, en deux ou plusieurs parties en parallèle et à n'utiliser qu'une partie pendant la mise en vitesse.

Le courant est ainsi notablement diminué et après couplage la réactance de l'alternateur-moteur est normale.

Le schéma est celui de la figure 9 pour un enroulement à deux voies en parallèle.

La séquence est simple: le disjoncteur principal $D^{1}$ est tout d'abord fermé, $D^{2}$ étant ouvert. La machine monte en vitesse et au voisinage du synchronisme, le disjoncteur (ou simple enclencheur) $D^{2}$ est fermé, mettant en service la deuxième partie de l'enroulement.

Il faut que le mode d'enroulement se prête bien à la division, en deux ou plusieurs voies en parallèle, sinon le procédé n'est pas intéressant. Il faut, également, vérifier que le couple de démarrage est suffisant pour accélérer la machine jusqu'au voisinage du synchronisme.

\section{3. - Démarrage par machine auxiliaire accouplée sur le même arbre}

\subsection{Démarrage par turbine hydraulique.}

Cette turbine peut être du type Francis ou Pelton. La turbine Pelton présente l'avantage de sa simplicité, mais compte-tenu de l'enfoncement important de l'usine nécessité par le fonctionnement en pompe, l'utilisation d'une Pelton pose le problème de l'évacuation de son débit.

$\mathrm{Ce}$ problème peut trouver trois solutions:

- évacuation gravitaire, si la topographie le permet;

- évacuation dans une excavation capable d'emmagasiner le volume d'eau utilisé pour un démarrage, ce qui implique d'avoir des moyens d'exhaure suffisamment dimensionnés; 


\section{G. MARTIN}

- fonctionnement en contre-pression, roue dénoyée dans l'air.

La turbine Francis présente souvent quelques difficultés d'implantation sur la ligne d'arbre. Par contre, fonctionnant normalement en contre-pression, elle peut être raccordée à l'aspirateur de la pompe-turbine sans difficultés majeures. Une variante qui a été proposée consiste à faire la restitution à travers l'arbre creux et le moyeu de la pompeturbine.

Si la turbine de lancement est située au-dessus de la pompe, la ligne d'arbre est allongée et le diamètre de l'arbre principal impose, pour la turbine de lancement, un diamètre de la roue relativement important. Si elle est endessous, il se pose alors le problème de la traversée de l'aspirateur de la pompe par l'arbre de la turbine de lancement.

Dans les deux cas le tracé des conduites d'amenée et de refoulement conduit à des dispositions de génie civil pouvant être onéreuses.

Toutefois, ce procédé de lancement offre quelques avantages: aucune sujétion particulière pour le moteur, pas d'à-coup sur le réseau, rusticité du système (surtout avec la turbine Pelton), couple maximal obtenu à vitesse nulle d'où un dimensionnement avantageux de la machine (sa puissance est inférieure à celle du moteur asynchrone correspondant).

Il faut noter, également, la possibilité de démarrer plusieurs groupes simultanément. Toutefois, ce procédé est surtout convenable économiquement pour une centrale n'ayant qu'un ou deux groupes de pompage de grande puissance, étant donné que la turbine de lancement montée sur l'arbre ne peut servir qu'à un seul groupe.

La figure 10 représente un exemple d'installation avec turbine Francis de lancement (Langenprozelten, Allemagne de l'Ouest).

\subsection{Démarrage par moteur asynchrone.}

Ce procédé qui est fréquemment employé consiste à monter, sur l'arbre du groupe, un moteur de lancement, moteur asynchrone à bagues alimenté par le réseau.

Le réglage de la vitesse s'effectue en agissant sur la résistance du rotor à l'aide d'un rhéostat de démarrage, liquide ou métallique.

Le couple maximal du moteur doit être légèrement supérieur au couple résistant maximal, pour permettre d'atteindre la vitesse de synchronisme et le couplage.

Pour éviter un dimensionnement prohibitif du moteur de lancement, le démarrage se fait, pratiquement, toujours roue dénoyée. A titre indicatif, le couple de barbotage de la roue dénoyée est d'environ 4 à $5 \%$ du couple nominal en pompe et la puissance du moteur est de l'ordre de 6 à $8 \%$ de la puissance maximale de pompage de la pompeturbine.

Les groupes 1,2 et 3 de Revin $(200 \mathrm{MW})$ sont équipés pour ce mode de démarrage.

L'alimentation du moteur de lancement par le réseau peut être réalisée de deux façons distinctes:

a) En parallèle (fig. 11 a).

C'est le montage le plus courant. Au démarrage, le moteur de lancement est alimenté par le réseau, côté M.T. du transformateur principal, directement à la tension du jeu de barres, ce qui est préférable, sinon par l'intermédiaire d'un transformateur ou par un réseau auxiliaire indépendant.

Deux façons de synchroniser sont possibles:

- si le moteur de lancement a le même nombre de paires de pôles que le groupe principal, en fin de démarrage l'alternateur-moteur est fermé sur le réseau par l'enclencheur sens «pompage » et s'accroche par le couple synchronisant (avec un léger à-coup de courant);

- si le moteur possède une paire de pôles de moins que l'alternateur-moteur, son réglage de vitesse se fait par le rhéostat de démarrage et la synchronisation au réseau par l'enclencheur est réalisée comme pour le couplage en turbine, à la vitesse de synchronisme.

b) En série (fig. n" $11 b$ ).

Les deux stators de l'alternateur et du moteur de lancement sont montés en série, moteur de lancement côté neutre de l'alternateur. Pour le démarrage le disjoncteur du groupe est enclenché, l'impédance de l'alternateur-moteur étant plus faible, la tension à ses bornes est plus réduite, l'accélération de l'ensemble est alors assurée par le moteur de lancement. Le courant absorbé est très limité. Pendant la mise en vitesse la tension aux bornes de la machine principale augmentant, si son circuit amortisseur le permet, elle contribue à la production du couple accélérateur. Dès que le glissement atteint sa valeur de régime asynchrone, le moteur principal est synchronisé par la fermeture de l'excitation et le moteur de lancement est court-circuité.

Cette alimentation en série est avantageuse pour des machines à couple résistant important en fin de démarrage.

Le procédé avec moteur de lancement présente les avantages suivants:

- surcharge négligeable pour le réseau;

- possibilité d'utiliser le moteur de lancement en frein pour l'arrêt du groupe, ou en alternateur excitateur débitant sur des redresseurs pour l'excitation de l'alternateurmoteur (toutefois, une source auxiliaire d'alimentation du rotor est alors nécessaire);

- possibilités de démarrage simultané de plusieurs groupes. Les inconvénients à mentionner sont:

- le coût élevé du moteur proprement dit et de son appareillage;

- l'obligation d'avoir un moteur par groupe;

- l'allongement de la ligne d'arbre;

- le schéma relativement compliqué (surtout s'il y a utilisation du moteur en frein ou en alternateur auxiliaire);

- le temps de démarrage assez long avec un moteur de dimensionnement raisonnable;

- le nombre de démarrages successifs limité.

\subsection{Démarrage par moteur courant continu.}

Le moteur de lancement peut être un moteur à courant continu pour lequel la vitesse est réglée, facilement, en agissant sur le flux inducteur ou sur la tension aux bornes de l'induit. Dans le cas du lancement des groupes réversibles, les puissances mises en jeu sont importantes. Si l'on ne dispose que de courant alternatif, il faut transformer ce dernier en courant continu.

Parmi les moyens les plus couramment utilisés, il faut citer le dispositif Ward-Léonard dans lequel le réglage de la vitesse est obtenu en faisant varier la tension d'alimentation en réglant l'excitation de la génératrice. Le schéma est celui de la figure 12 .

Il faut remarquer que ce dispositif Ward-Léonard qui peut assurer successivement le démarrage de plusieurs groupes présente l'avantage de réaliser des démarrages très progressifs, tout en ne nécessitant que des moteurs et du matériel auxiliaire très classiques.

$\mathrm{Ce}$ dispositif peut, également, être intéressant si l'on utilise l'excitatrice principale de l'alternateur-moteur comme moteur de lancement, à condition de la dimensionner en conséquence. 
(01)

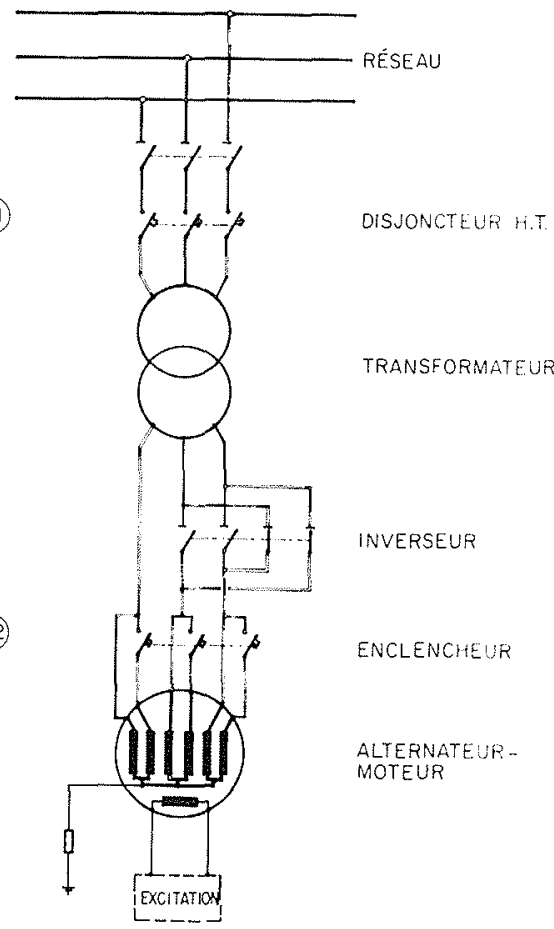

91

9/ Démarrage en asynchrone avec tension réduite par fractionnement de l'enroulement statorique.

10/ Démarrage par turbine hydraulique accouplée sur le même arbre (d'après Water Power, janvier 1972, usine de Langenprozelten).

11 a/ Démarrage par moteur asynchrone. Moteur alimenté en parallèle sur les barres M.T.

$11 \mathrm{~b} /$ Démarrage par moteur asynchrone. Moteur alimenté en série avec l'alternateur moteur.

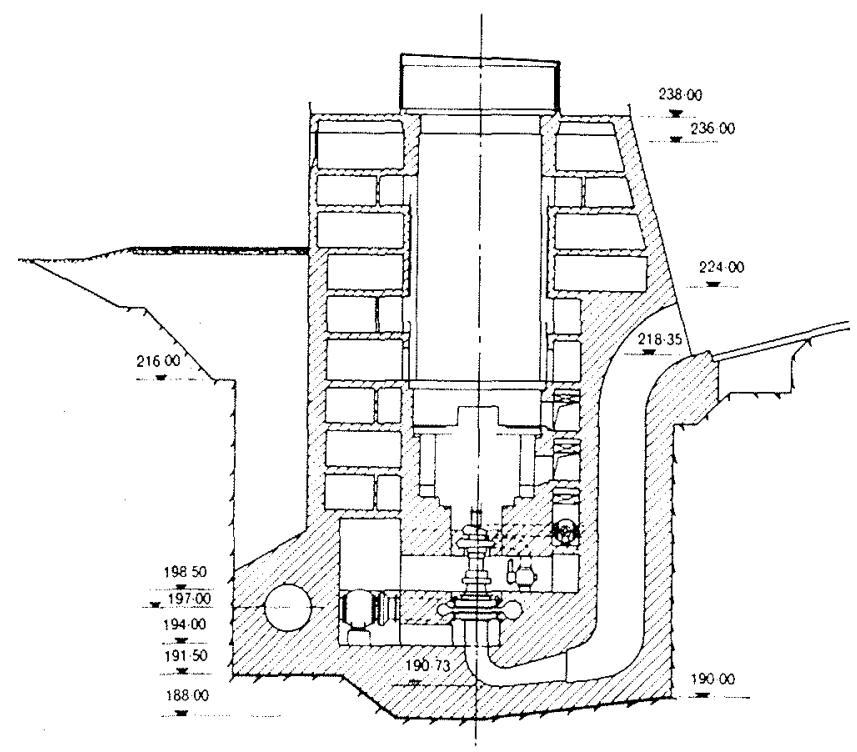

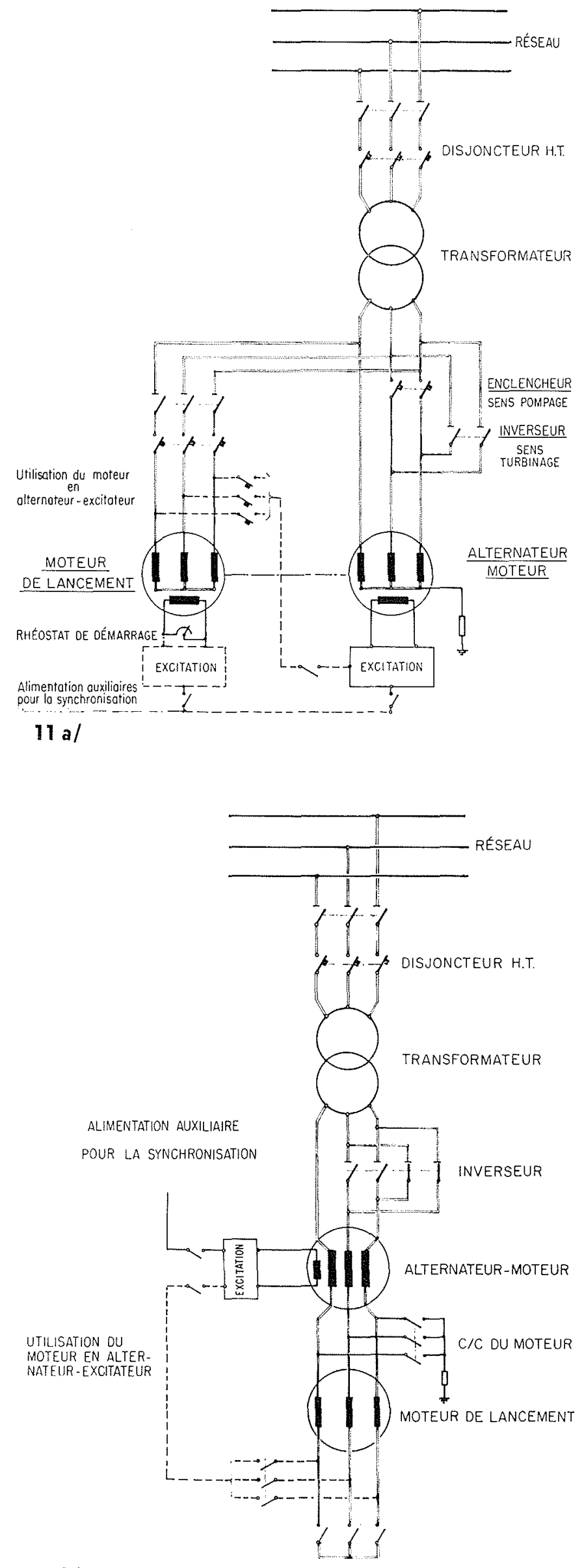

$11 \mathrm{~b} /$ 


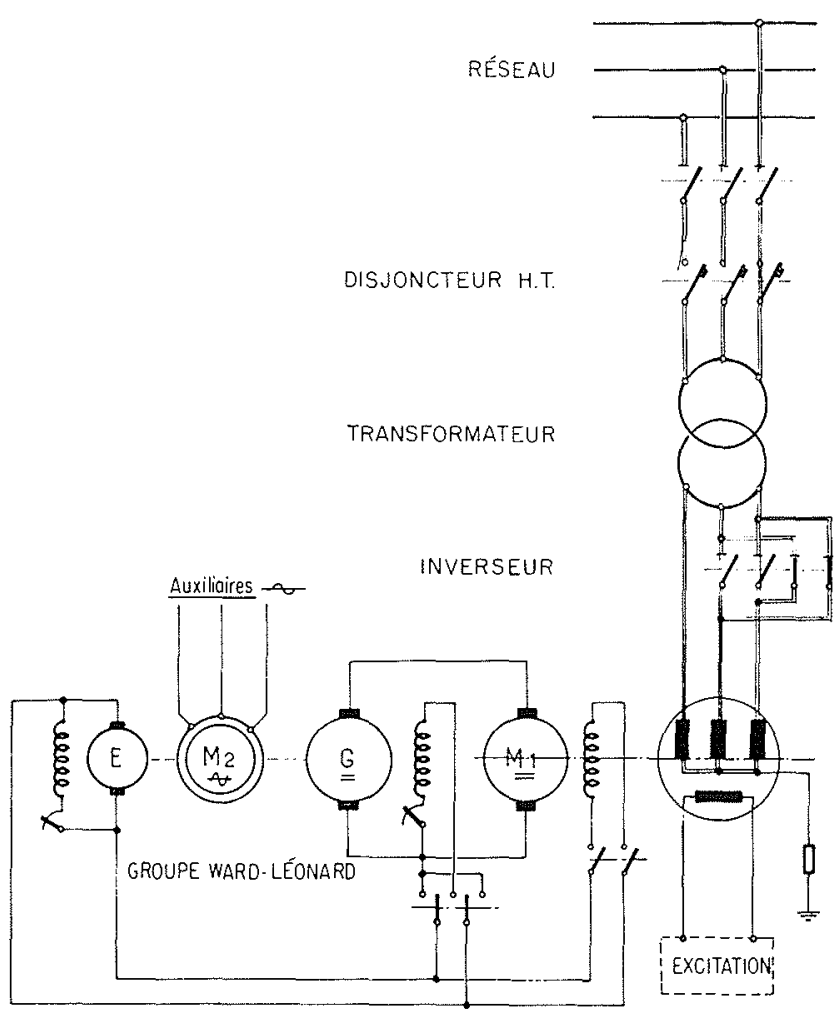

12/ Démarrage par moteur courant continu. Moteur alimenté par groupe Ward-Léonard.

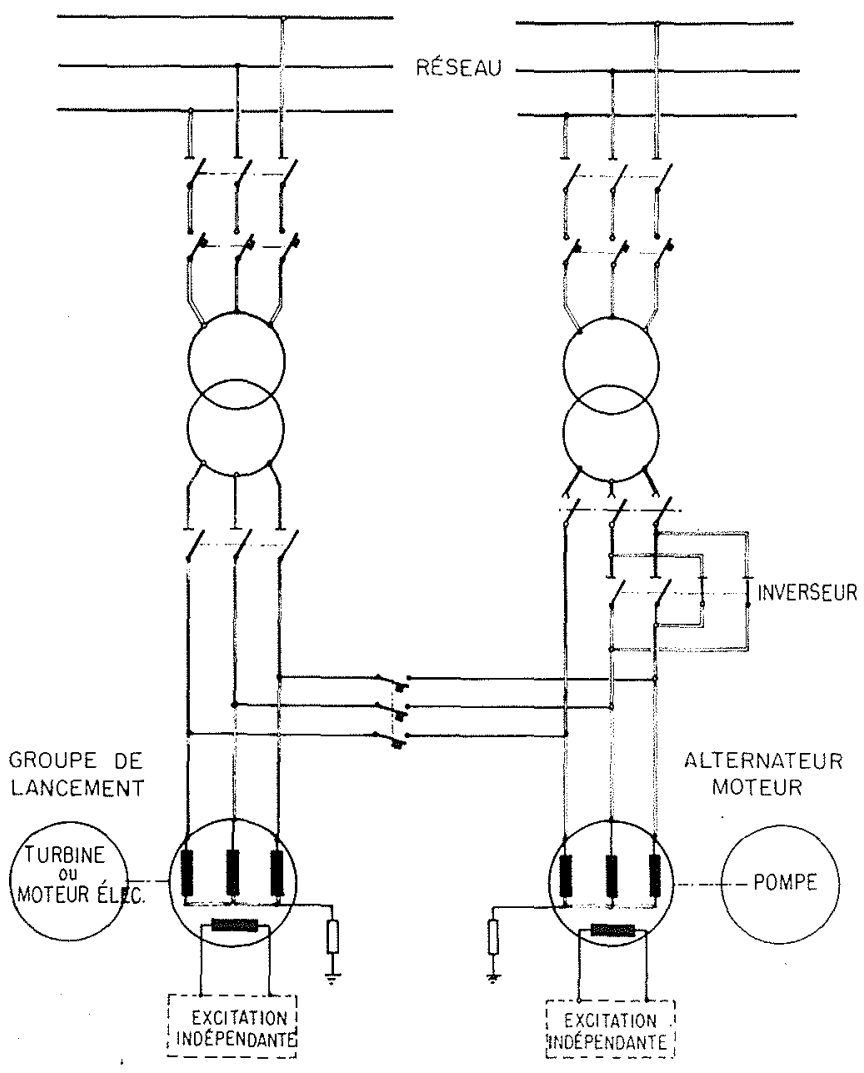

13/ Démarrage à fréquence variable avec groupe séparé " dos à dos ». Méthode synchrone ou asynchrone-synchrone.
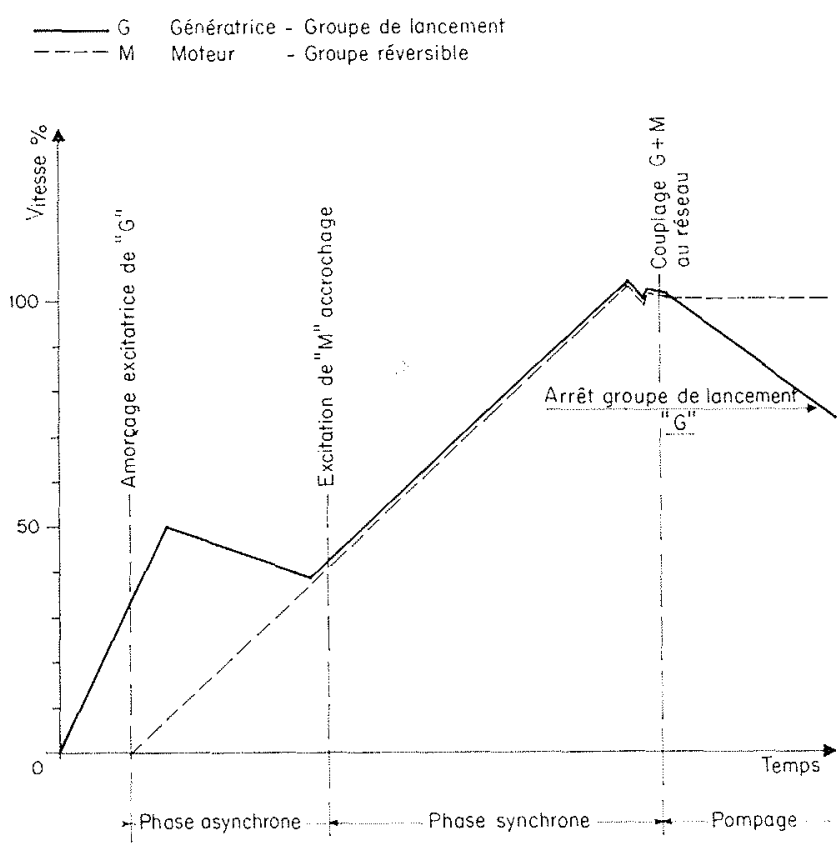

14/ Démarrage à fréquence variable - Méthode mixte : asynchronesynchrone - Courbes de mise en vitesse.

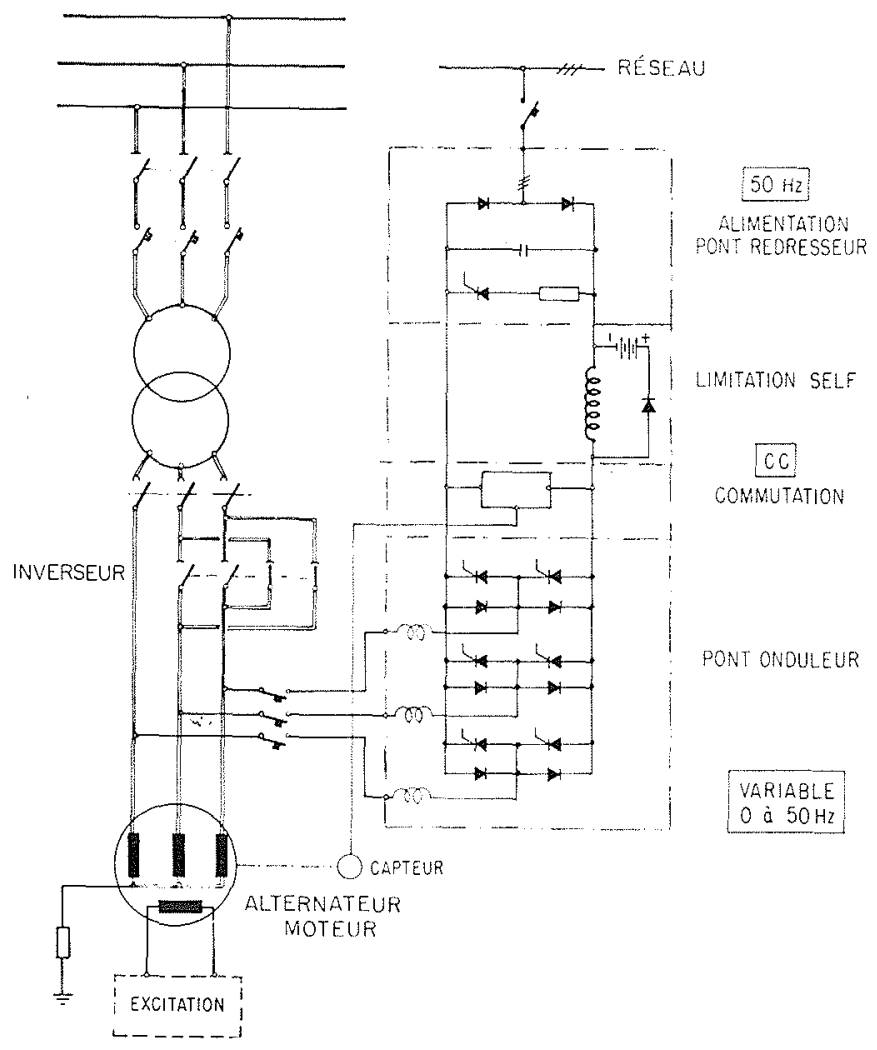

15/ Démarrage à fréquence variable avec convertisseur statique. 


\section{4. - Démarrage ò̀ fréquence variable}

Le principe du lancement des groupes à fréquence variable consiste à utiliser une source auxiliaire, commune à tous les groupes de l'installation et dont la fréquence peut varier, suivant une loi bien définie, de 0 à la fréquence nominale.

Cette source auxiliaire peut être, soit un groupe rotatif séparé à entraînement hydraulique ou électrique, soit un convertisseur statique; quelle que soit la source, elle est le plus souvent unique pour une usine, il n'y a donc pas de possibilité de démarrage simultané de plusieurs groupes. De plus, dans cette hypothèse, une panne sur la source rend indisponible toute l'usine.

\subsection{Démarrage avec groupe séparé (dos à dos).}

\subsubsection{CONSTITUTION DU GROUPE DE LANCEMENT.}

Il est possible d'utiliser :

a) Un groupe séparé hydraulique constitué par un alternateur entraîné par une turbine Pelton ou Francis, spécialement dimensionné pour le lancement. Il peut être valorisé par son utilisation comme groupe d'alimentation des services auxiliaires de l'usine (en dehors des périodes de mise en service du pompage), ou comme groupe d'appoint de puissance en turbine en heures de pointe. Dans ces conditions, il peut être largement dimensionné et permettre ainsi le démarrage roue noyée, ce qui est une simplification intéressante.

Il est également possible d'envisager que dans une usine à « $n$ 》groupes réversibles, l'un quelconque puisse être utilisé en groupe lanceur, mais la capacité de pompage de l'usine n'est plus alors que de $(n-1)$ groupes. Ceci est d'ailleurs parfaitement admissible dans la plupart des installations, la puissance demandée en turbine étant supérieure à la puissance de pompage. La fiabilité est meilleure, la marche de l'usine en pompage n'étant tributaire que d'un seul et unique groupe.

Le procédé avec groupe séparé à entraînement hydraulique est économique mais le bilan dépend essentiellement des conditions d'installation.

Par contre, l'intégration du groupe séparé hydraulique dans le génie civil peut présenter quelques difficultés en raison de l'enfoncement nécessaire aux usines à groupes réversibles. Il peut être quelquefois intéressant d'installer le groupe hors de l'usine elle-même, mais à proximité.

Il peut, également, être envisageable d'utiliser un groupe existant dans une centrale voisine et relié par une ligne directe à la centrale de pompage.

b) Un groupe séparé électrique dont l'alternateur est entraîné par un moteur électrique, en principe asynchrone, équipé d'un rhéostat de démarrage permettant de contrôler sa vitesse. L'installation est facile car il n'y a pas de sujétions d'ordre hydraulique, mais l'un des intérêts de la solution précédente - l'utilisation en pointe du groupe auxiliaire disparaît.

\subsubsection{Méthode de mise en VItesse.}

La mise en vitesse du groupe principal à l'aide du groupe séparé, hydraulique ou électrique, peut se faire suivant deux méthodes :

- méthode entièrement synchrone;

- méthode mixte: asynchrone - synchrone.

Dans les deux méthodes, mais en particulier dans la méthode synchrone, la difficulté la plus importante consiste à obtenir la puissance suffisante pour vaincre le couple de décollage. Pratiquement l'injection d'huile au pivot est toujours indispensable.

Dans les deux cas il est souhaitable que la liaison électrique, entre les deux machines, soit aussi courte et aussi peu impédante que possible.

a) Méthode synchrone.

Le schéma de principe est le suivant (fig. 13) : les stators des machines sont couplés entre eux électriquement et les rotors sont excités par une ou des sources d'excitation indépendante (groupes rotatifs ou convertisseurs statiques) pour pouvoir fournir le maximum de puissance d'excitation dès le démarrage et obtenir, par conséquent, le couple électrique maximal sur le moteur.

Le groupe auxiliaire ou groupe de Jancement est démarré lentement pour obtenir plus facilement l'accrochage. Le groupe principal décolle, l'ensemble est alors en régime asynchrone pendant un temps très court. Le glissement diminue et, à faible puissance, les deux machines se synchronisent et passent en régime synchrone à fréquence variable. Le groupe de lancement accélère, le groupe principal suit jusqu'à la fréquence nominale. Après ajustage de la tension, l'ensemble peut être couplé au réseau. Le groupe de lancement est alors déclenché et mis à l'arrêt. La charge est prise, comme dans les autres cas, par ouverture du vannage ou de la vanne de refoulement de la pompe.

Ce procédé présente l'avantage de ne provoquer aucun à-coup sur le réseau ni contrainte sur l'alternateur-moteur et d'être utilisable sans difficultés particulières pour des unités de grande puissance.

En contrepartie, il faut noter l'inconvénient principal de la méthode: la nécessité d'avoir des groupes équipés de sources d'excitation indépendante.

b) Méthode mixte: asynchrone-synchrone.

Cette méthode est une combinaison de la méthode asynchrone directe avec la méthode synchrone pure, exposée au paragraphe précédent.

Le schéma de principe est identique au précédent, mais sans les dispositifs d'excitation séparée.

Les stators sont couplés électriquement, mais le rotor de l'alternateur-moteur n'est pas excité. La génératrice de lancement est montée rapidement en vitesse, jusqu'à environ 40 à $50 \%$ de sa vitesse nominale. Au passage à la vitesse d'amorçage de son excitation, la tension apparaît et le moteur démarre en asynchrone. A la vitesse prédéterminée, la génératrice est ralentie tandis que le moteur continue son accélération. A l'égalité des vitesses le moteur est excité et il se synchronise avec la génératrice. La phase synchrone se déroule alors comme dans le cas du démarrage synchrone. Les courbes de la figure 14 schématisent le processus.

Cette méthode possède tous les avantages de la méthode synchrone pure, mais elle permet de s'affranchir de son inconvénient majeur: l'obligation d'avoir des sources d'excitation séparées.

Par contre, elle impose l'obligation de dimensionner le circuit amortisseur, pour supporter l'échauffement pendant la période asynchrone du démarrage, ce qui est inutile avec la méthode synchone pure.

Le groupe réversible n" 4 de l'usine de Vouglans dans le Jura est démarré suivant cette méthode à l'aide du groupe classique voisin $n^{\circ} 3$.

\subsection{Démarrage avec convertisseur statique de fréquence.}

Le groupe séparé mentionné ci-dessus, peut être remplacé par un convertisseur statique à fréquence variable (fig. 15) permettant, à l'aide d'une source alternative à fréquence fixe, d'obtenir une tension à une fréquence variable. 


\section{G. MARTIN}

Le principe consiste à redresser le courant alternatif du réseau, puis, avec le courant continu obtenu, alimenter un onduleur qui fournit le courant à fréquence variable nécessaire.

L'ensemble de l'appareillage comprend schématiquement : - un pont-redresseur;

- un dispositif de limitation;

- un circuit de commutation;

- un pont-onduleur.

Par le dispositif de commutation la fréquence de sortie varie progressivement de 0 à $50 \mathrm{~Hz}$. L'alternateur-moteur démarre en «synchrone » et à la vitesse de synchronisme celui-ci est couplé au réseau. Le convertisseur est alors disponible pour démarrer un autre groupe.

Ce procédé de démarrage par convertisseur de fréquence présente l'avantage de permettre d'obtenir des couples moteurs relativement élevés, mais nécessitant, toutefois, le dénoyage de la roue et linjection d'huile. Il n'est valable, économiquement, que pour une usine à plusieurs groupes pour lesquels des temps de démarrage assez longs (10 à $15 \mathrm{mn}$ ) sont admissibles.

Il est fiable et ne demande qu'un entretien réduit. Théoriquement tout au moins, la puissance n'est pas limitée.

\section{5. - Démarrage par modification du circuit hydraulique}

\subsection{Modification du circuit de l'installation.}

Dans ce mode de démarrage, c'est la pompe elle-même qui est utilisée en «moteur de lancement » en réalisant un circuit hydraulique dans le sens pompage $(*)$.

(*) Demande de brevet E.D.F. en cours.
Il n'est pas pensable que ce mode de démarrage (essentiellement fonction des caractéristiques de chute et des conditions générales de l'aménagement) puisse permettre d'amener le groupe à sa vitesse nominale. Mais un tel procédé peut, dans certains cas favorables, faciliter les démarrages des groupes réversibles.

Deux systèmes peuvent être imaginés :

\subsubsection{UTILISATION DE L'EAU DU BASSIN INFÉRIEUR (voir schéma fig. $16 a$ ).}

La vanne (2) sur l'aspirateur de la pompe est ouverte. La vanne (1) sur le refoulement est fermée.

Une vanne « de lancement » (3) permet de faire circuler l'eau dans le sens des flèches et de l'évacuer dans un bassin vidangeable par pompage (ou gravitairement si les conditions topographiques locales le permettent).

Lorsque la vitesse d'équilibre, fraction de la vitesse nominale, est atéeinte, la vanne de lancement est fermée, le démarrage peut être poursuivi en asynchrone par exemple.

Les avantages de ce système sont les suivants :

- les vannes d'aspiration et de refoulement ainsi que le corps de pompe ne sont pas soumis à aucune pression anormale résultant du système proposé;

- l'automatisme est simple et la sécurité facile à assurer. Le système n'impose qu'une seule vanne de lancement qui, en marche, supporte la pression totale, mais qui peut ne pas avoir à assurer le plein débit.

Ce système semble particulièrement convenir pour les cas de hautes chutes avec enfoncement important de la pompe et de grand marnage sur le bassin inférieur.

Par contre il y a quelques inconvénients:

- le couple moteur disponible est très limité;

- le problème d'évacuation de l'eau utilisée au bassin inférieur avec pompage, ou évacuation gravitaire, se pose comme d'ailleurs dans le cas du démarrage par turbine.

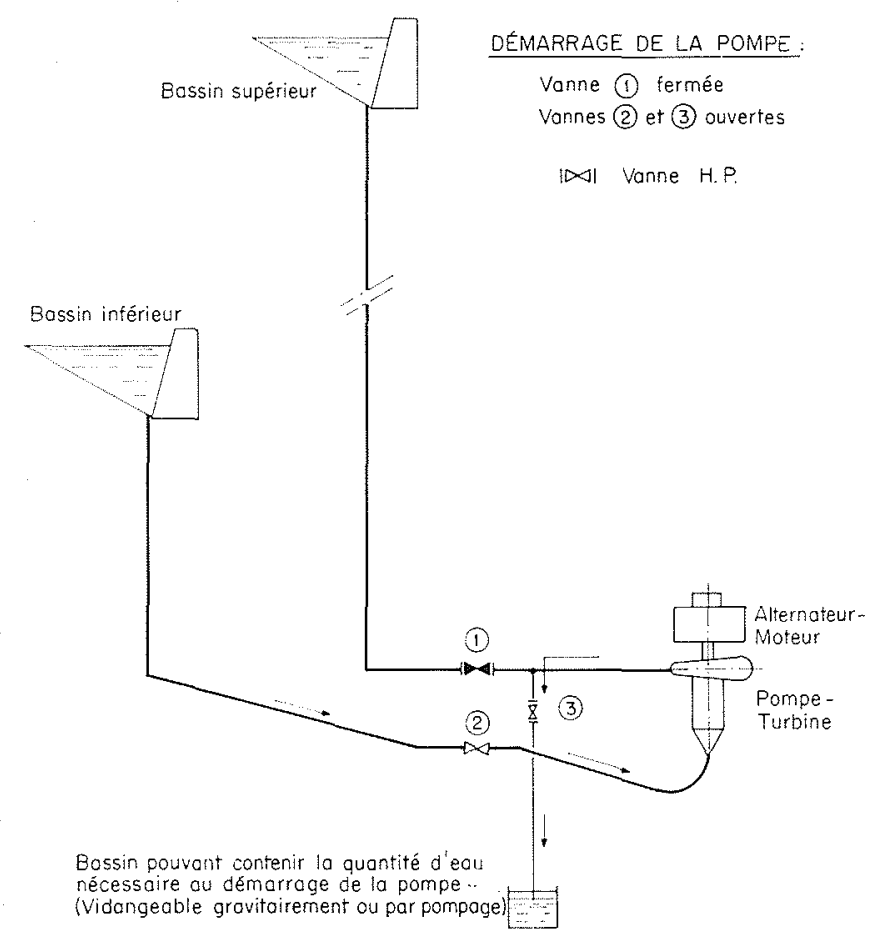

16 a/ Démarrage par modification du circuit hydraulique. Utilisa tion de l'eau du bassin inférieur.

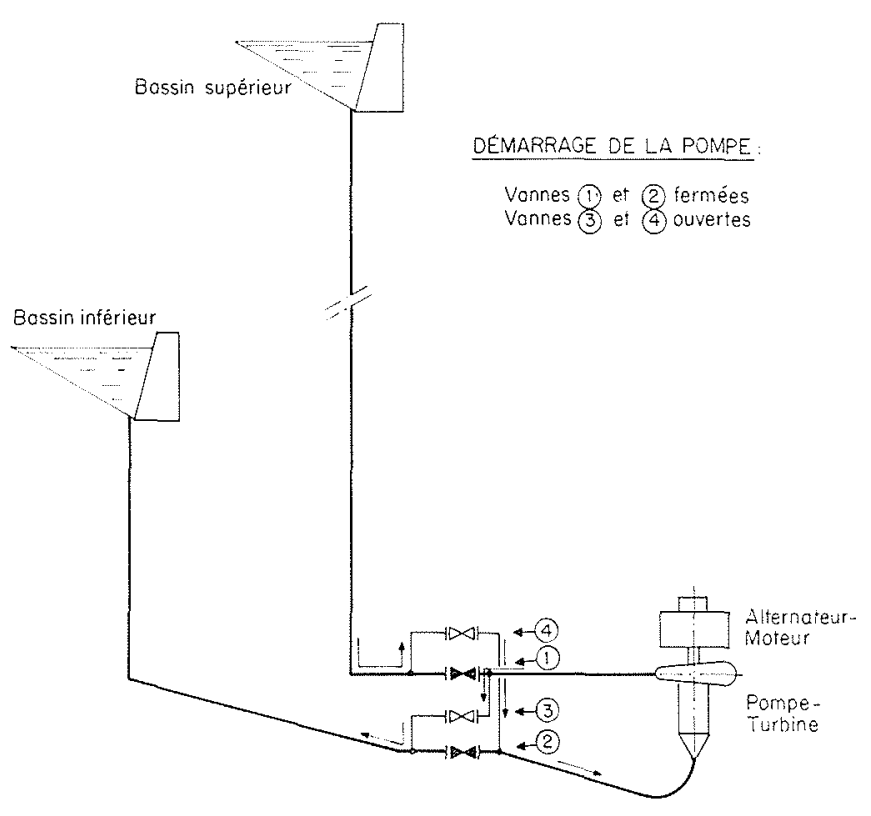

$16 \mathrm{~b} /$ Démarrage par modification du circuit hydraulique. Utilisation de l'eau du bassin supérieur. Le corps de la pompe supporte la pleine pression. 
5.1.2 UTILISATION DE L'EAU DU BASSIN SUPÉRIEUR (voir schéma fig. $16 b$ ).

Les vannes sur l'aspirateur (2) et sur le refoulement (1) sont fermées. A l'aide d'un jeu de deux vannes auxiliaires (3) et (4), l'eau du bassin supérieur est dirigée sur le collecteur d'aspiration de la pompe et le collecteur de refoulement de celle-ci est mis en communication avec le bassin inférieur.

\section{- Avantages:}

- couple moteur important;

- pas de problème d'évacuation d'eau.

- Inconvénients :

- il faut deux vannes de lancement (vannes qui doivent pouvoir résister à la pleine pression);

- schéma d'automatisme plus compliqué que dans la méthode avec utilisation de l'eau du bassin inférieur;

- le circuit d'aspiration de la pompe doit supporter la pleine pression, ce qui renchérit la machine, sinon il faut prévoir un détendeur, organe délicat et coûteux, ce qui complique le schéma.

\subsection{Modification du circuit hydraulique de la turbine.}

Il s'agit essentiellement de la pompe-turbine "isogyre » des Ateliers des Charmilles.

Cette machine est constituée d'une bâche spirale unique, raccordée au réservoir supérieur et de deux roues accolées: l'une de turbine, l'autre de pompe. Une double vannefourreau intégrée permet de choisir le mode de fonctionnement en alimentant soit le côté pompe, soit le côté turbine (voir coupe fig. 17). Les deux conduits d'évacuation en sortie de la roue turbine et d'aspiration en entrée de la roue pompe sont raccordés au bassin inférieur.

Nous ne mentionnerons que pour mémoire ce type de machine, car elle ne constitue pas réellement un groupe "réversible » puisque l'un de ses avantages essentiels est son sens de rotation unique en turbine et en pompe.

Le démarrage en pompe qui est assuré normalement par la turbine, la roue pompe étant dénoyée, ne pose aucun problème particulier. Après couplage au réseau la roue de la pompe est renoyée et par le jeu des vannes-fourreau la circulation s'établit dans le sens «pompage». Le retour à la marche en turbine s'effectue tout aussi simplement et rapidement.

La disposition peut être à axe vertical ou horizontal (fig. 18)

A notre connaissance, il n'existe actuellement qu'un groupe de ce type, à axe horizontal, d'une puissance de $10 \mathrm{MW}$ à Robiei en Suisse.

\section{6. - Conclusion}

Ainsi que nous venons de le voir, le choix de la meilleure méthode de démarrage d'un groupe réversible n'est pas simple. Il dépend de nombreux critères, mais plus particulièrement :

- des caractéristiques propres au groupe à lancer: puissance, inertie, couple-moteur et couple-résistant, réactances, constantes de temps...;

- des caractéristiques du réseau: puissance de courtcircuit, à-coup de courant et chute de tension admissibles, dimensionnement des lignes et des transformateurs de réseau éventuels;

- des temps que l'on peut admettre pour le changement de marche (turbine en pompe et vice-versa);

- du nombre de groupes nécessaires en turbine et en pompe (par exemple si l'on a besoin de (n) groupes en

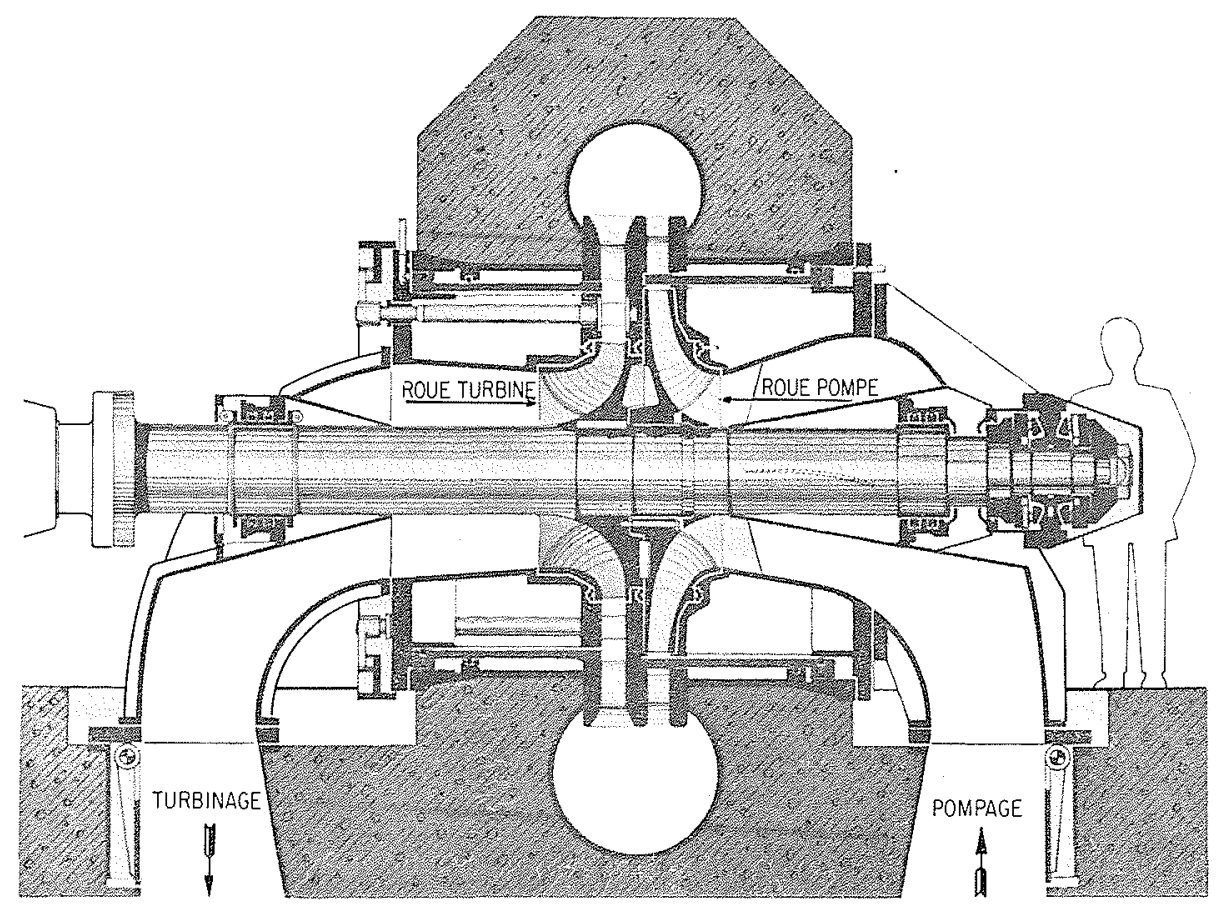

17/ Turbine isogyre Charmilles. 


\section{G. MARTIN}

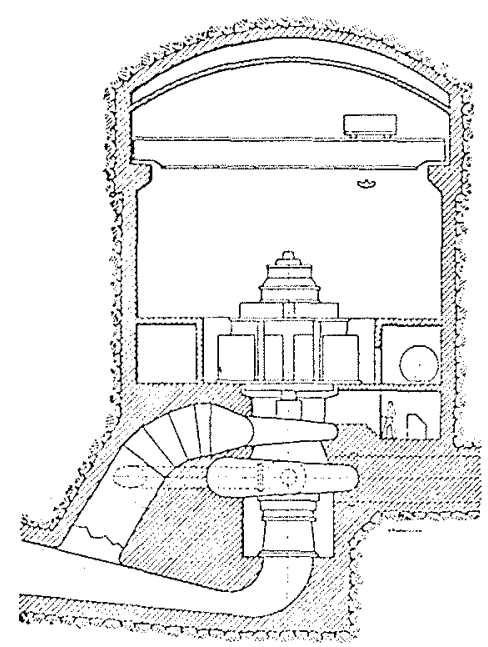

Disposition ventical

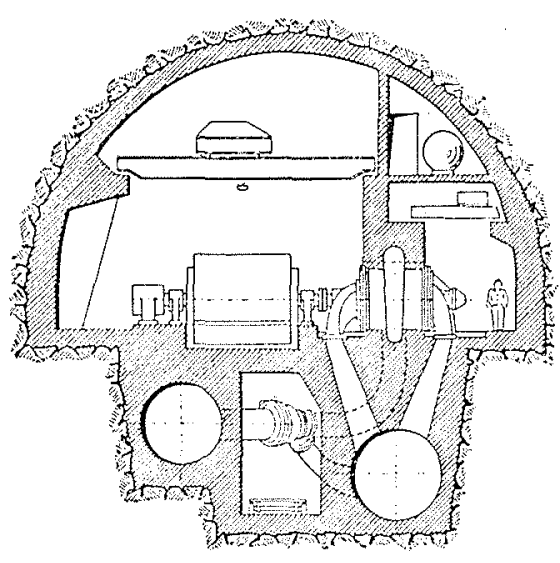

Disposition horizontale

18/Turbine isogyre Charmilles. Dispositions d'installation.

turbine et seulement de $(n-1)$ en pompe, le procédé s'impose tout naturellement);

- des conditions locales particulières, telles que: facilité d'installation d'un groupe spécial ou possibilités de raccordement à un groupe d'une usine voisine au lancement dos à dos par exemple.

La méthode la plus simple et la plus économique lorsqu'elle est techniquement possible - est le démarrage synchrone direct, mais la tendance actuelle de plus en plus marquée vers l'augmentation de la puissance unitaire des groupes réversibles risque, dans l'avenir, de poser des problèmes, non seulement d'ordre technologique, mais également d'exploitation des réseaux et peut éventuellement conduire à adopter un autre procédé.

Dans ce cas, le démarrage à tension réduite peut souvent apporter une solution intéressante. Le procédé est économique (en particulier celui avec réduction de tension par bobine de réactance). Il s'adapte bien aux machines rapides à faible réactance au démarrage, mais il ne peut être utilisé, valablement, qu'avec des machines à couple résistant faible.

Le moteur asynchrone de lancement, monté en bout d'arbre, s'adapte bien à la plipart des cas, mais cette solution est chère, surtout si l'usine est équipée de plusieurs groupes.

Toutes les autres méthodes ne sont valables que dans des cas plus spéciaux. Notons toutefois que le convertisseur statique peut, avec les progrès de la technique, devenir compétitif pour des usines à nombreux groupes.

En conclusion, nous pouvons dire qu'aucune méthode n'est parfaite et que, pour un même problème, plusieurs solutions sont possibles.
M. le Président remercie M. MARTiN de son intéressant exposé; les nombreuses possibilités qu'il a évoquées pour le démarrage des groupes laissent entrevoir la difficulté de choisir le système le mieux adapté à chaque cas particulier.

M. le Président ouvre ensuite la discussion.

Commentant une série de diapositives, M. Piguet (Charmilles, Genève) donne les informations suivantes sur l'évolution et l'état actuel des machines « isogyres ».

Charmilles a poursuivi le développement des isogyres; il s'agit d'une machine qui, dans un encombrement très réduit, rassemble les avantages d'un groupe ternaire. Ceux-ci sont essentiellement: démarrage avec la turbine, sens de rotation unique, tracé le plus favorable des roues de pompe et de turbine, dont les caractéristiques peuvent être choisies en toute liberté, hautes performances de rendement. Le développement a porté essentiellement sur les tracés hydrauliques qui ont été étudiés en laboratoire et sur le groupe de Robiei (chute $390 \mathrm{~m}$, puissance en turbine $10 \mathrm{MW}$, en pompe $8,2 \mathrm{MW}, 1500 \mathrm{tr} / \mathrm{mn}$ ) puis sur des dispositifs de construction qui font l'objet de brevets. Notons, en particulier, les évacuateurs, à

\section{Discussion}

la sortie du joint central qui protègent la roue dénoyée de toute projection d'eat et les labyrinthes à jeu variable qui dispensent de toute injection d'eau de refroidissement sur la roue dénoyée.

Les résultats des mesures de rendement de la machine industrielle de Robiei sont ceux que l'on peut attendre d'une turbine et d'une pompe conventionnelles, fonctionnant avec les mêmes caractéristiques hydrauliques.

M. Fauconnet (Charmilles, Genève) apporte, en s'aidant d'une série de diapositives, d'intéressantes précisions sur les performances mesurées sur le groupe isogyre de Robiei.

Lors des premiers essais de ce groupe en 1968, sous une chute de $335 \mathrm{~m}$, le rendement maximum obtenu en turbine, mesuré par la méthode thermodynamique, a été de $90,8 \%$; ce chiffre tient compte des pertes de la roue de pompe dénoyée; les fuites au joint central étaient éliminées par le dispositif chasse-gouttes évoqué par M. PIGUET mais le perfectionnement conduisant à supprimer l'arrosage des labyrinthes n'était pas encore installé.

Le groupe de l'usine de Bouvantes (E.D.F.) de vitesse spécifique et de dimensions proches de celui de Robiei, mais qui fonctionne 
sous une chute environ moitié moindre, a donné en turbine un rendement maximum de $91,0 \%$.

Les dégâts subis par l'ouvrage d'amenée de Robiei ont nécessité une longue mise hors service de cette centrale. Au cours de cette période l'isogyre a été démontée pour contrôle. Les joints hydrauliques ont subi une légère retouche.

Les essais du groupe isogyre se sont poursuivis en 1970, dès la remise en exploitation de la centrale. Ils ont eu lieu sous quatre chutes réparties dans le domaine prévu d'utilisation $(276 \mathrm{~m}<\mathrm{H}$ $<400 \mathrm{~m}$ ).

En marche turbine, ces essais font apparaitre un rendement maximum $o=90,0 \%$ sous $340 \mathrm{~m}$. L'écart de $-0,8 \%$ avec les résultats de 1958 montre l'influence du jeu radial du labyrinthe sur le rendement. Par ailleurs, les performances constatées coïncident, sur tout le domaine d'utilisation, avec celles d'une turbine classique de même vitesse spécifique.

M. FauconNet montre ensuite les caractéristiques « débit-chute» et la courbe de rendement pour le fonctionnement en pompe obte- nue d'une part sur un modèle, et d'autre part sur la machine industrielle. La comparaison des rendements maximaux ainsi mesurés avec ceux relevés sur des modèles de pompe - de construction suisse - de même vitesse spécifique, montre le fonctionnement tout à fait satisfaisant des isogyres en pompe également.

M. Amblard signale un autre mode de démarrage dont il n'a pas encore été parlé au cours de cette séance. 11 consiste à "renverser 》 les directrices de façon que leur orientation impose un écoulement tel que la roue soit entrainée « en turbine » dans le sens pompe.

Si lon n'atteint ainsi que 80 à $90 \%$ de la vitesse de synchronisme, on poursuit le démarrage en utilisant, à cet effet, l'alternateur comme moteur.

Sur cette question de M. Martin, M. Amblard précise qu'il ne connaît pas d'application pratique de ce procédé mais que des essais ont été effectués en Allemagne.

Constatant avec regret que personne ne demande plus la parole, $M$. le Président clôt la discussion et lève la séance à 18 heures.

\section{Abstract \\ Pros and cons of various pumpwise start-up techniques for reversible sets}

Compared with ternary sets comprising two distinct conventional machines, i.e. a pump and a turbine, reversible pump-turbine units permit considerable reduction in the capital cost of pumped storage schemes provided power demands for pumpwise and turbinewise operation are similar and provided also that time of transition between modes is not a decisive factor.

On the other hand, reversible sets rotating either way pose several problems, a major one being the best way to effect pumpwise start-up, more especially when unit power is high.

A number of possible procedures are examined and, in each case, advantages, disadvantages and application constraints are stated.

Start-up procedures available may be classified as in Figure 1 (table).

From the turbine standpoint, start-up may be carried out with the runner either wet or dry. Wet runner start-up is clearly the simpler of the two, although high starting torque is required to overcome the drag of the water. Dry startup has the disadvantage of requiring expensive compressed-air equipment for dewatering the runner.

When unit power is large, the majorty of the start-up procedures call for a dry runner.

The curves of Figure 2 show the general shape of the variation of pump-turbine drag torque with speed of rotation for wet and dry runners. These curves also point up the advantages of supplying oil at the thrust bearing.

Choice between wet and dry modes depends largely on torque available and torque required.

As for the choice of the best start-up procedure for a reversible unit, this is by no means a simple matter since it depends on a large number of criteria, the nost important being as follows: (i) Characteristics of the unit to be started up, i.e. power, inertia, driving couple, drag couple, reactance, time, constants, etc.

(ii) Characteristics of the network : short-circuit power, acceptable voltage drop, line and transformer design, elc.

(iii) Times allowed for changing from pumping to generation and vice versa.

(iv) Number of units required for turbine and pump operation.

(v) Local conditions on site e.g. ease of installation of a special unit or possibility of connecting up with a unit in a neighbouring plant suitable for back-to-back start-up.

When technically feasible, direct synchronous start-up is the simplest, most economical procedure. However, the present trend towards increased reversible-unit power seems likely to create problems. These will be concerned not only with the design and construction of the machines but also with network operation. Such problems may lead to preferential use of other start-up procedures.

Start-up under reduced voltage may sometimes have advantages. This is an economical method (especially when reactance coils are used for voltage reduction) which is well suited to fast machines with low start-up reactance. However, use of this method should be confined to machines with low drag torques.

Asynchronous start-up motors on the same shaft are suited to most cases. However, this is an expensive solution especially if the plant is made up of several sets.

None of the other methods are valid except in rather special cases. However, it is worth noting that progress with the static converter may make this technique competitive with others in cases where schemes use large numbers of sets.

No method is perfect and for a given problem several solutions are possible. 